\title{
Email Based Verification of Data Storage in Cloud Computing
}

\author{
Dong Hyun Youm1), Anand Prakash2)
}

\begin{abstract}
Cloud computing gives an adaptable and helpful route for information sharing, which brings different advantages for both the general public and people. However, there exists a characteristic resistance for clients to straightforwardly outsource the mutual information to the cloud server since the information frequently contain significant data. In this manner, it is important to put cryptographically upgraded get to control on the mutual information. Personality based encryption is a promising cryptographical primitive to manufacture a pragmatic information sharing framework. Be that as it may, get to control is not static. That is, the point at which some client's approval is lapsed, there ought to be a component that can evacuate him/her from the system. Thusly, the disavowed client can't get to both the beforehand and along these lines shared information. To this end, we propose an idea called revocable-capacity character based encryption (RS-IBE), which can give the forward/in reverse security of ciphertext by presenting the functionalities of client disavowal and ciphertext redesign at the same time. Moreover, we display a solid development of RS-IBE, and demonstrate its security in the characterized security show. The execution examinations demonstrate that the proposed RS-IBE scheme has points of interest as far as usefulness and proficiency, and in this manner is achievable for a reasonable and savvy information sharing framework. At last, we give usage aftereffects of the proposed plan to exhibit its practicability.
\end{abstract}

Keywords : cloud computing, data sharing, revocation, identity-based encryption, ciphertext update, decryption key exposure.

\section{Introduction}

Cloud computing is a world view that gives huge computation limit and immense memory space requiring little to no effort [1]. It empowers clients to get planned administrations regardless of time and area over various stages (e.g., cell phones, PCs), and in this way conveys extraordinary comfort to cloud clients. Among various administrations gave by distributed computing, distributed storage administration, for example, Apple's iCloud [2], Microsoft's Azure [3] and Amazon's S3 [4], can offer a more adaptable and simple approach to

Received(April 8, 2016), Review Result(1st: April 25, 2016, 2nd: May 20, 2016), Accepted(June 10, 2016)

${ }^{1}$ Department of Applied Computer Engineering, Dankook University, 152 Jukjeon-ro, Suji-gu, Gyeonggi-do email: daivid@gmail.com

${ }^{2}$ (Corresponding Author) Department of Computer Science and Engineering, KL University email: prakash@gmail.com 
share information over the Internet, which gives different advantages to our general public [5], [6]. Nonetheless, it likewise experiences a few security dangers, which are the essential worries of cloud clients [7-12]. Firstly, outsourcing information to cloud server suggests that information is out control of clients. This may bring about clients' delay since the outsourced information for the most part contain significant and touchy data. Also, information sharing is frequently executed in an open and threatening environment, and cloud server would turn into an objective of assaults. Far and away more terrible, cloud server itself may uncover clients' information for illicit benefit. Thirdly, information sharing is not static. That is, the point at which a client's approval gets lapsed, he/she ought to no longer have the benefit of getting to the already and along these lines shared information. Therefore, while outsourcing data to cloud server, users also want to control access to these data such that only those currently authorized clients can share the outsourced data.

\section{Proposed System}

\subsection{Related Work}

\subsubsection{Revocable identity-based encryption}

The idea of identity-based encryption was presented by Shamir [13], and helpfully instantiated by Boneh and Franklin [14]. IBE dispenses with the requirement for giving an public key infrastructure (PKI)). Notwithstanding the setting of IBE or PKI, there must be a way to deal with renounce clients from the framework when essential, e.g., the expert of some client is lapsed or the mystery key of some client is revealed. In the conventional PKI setting, the issue of repudiation has been all around considered [15-19], and a few methods are broadly endorsed, for example, testament renouncement list or attaching legitimacy periods to authentications. Be that as it may, there are just a couple contemplates on repudiation in the setting of IBE.

Boneh and Franklin [14] initially proposed a characteristic denial route for IBE. They affixed the present era to the ciphertext, and non-denied clients occasionally got private keys for every era from the key expert. Shockingly, such an answer is not adaptable, since it requires the key expert to perform direct work in the quantity of non-disavowed clients[15-17]. What's more, a protected channel is basic for the key expert and non-denied clients to transmit new keys[18][19]. To vanquish this issue, Boldyreva, Goyal and Kumar [20] acquainted a novel 
approach with accomplish proficient denial. They utilized a double tree to oversee character with the end goal that their RIBE conspire lessens the intricacy of key repudiation to logarithmic (rather than straight) in the greatest number of system clients. Be that as it may, this plan just accomplishes particular security. Hence, by utilizing the previously mentioned repudiation system, Libert and Vergnaud [21] proposed an adaptively secure RIBE conspire in view of a variation ofWater's IBE plot [22], Chen et al. [23-26] developed a RIBE conspire from grids.

\subsubsection{Forward-secure cryptosystems}

In 1997, Anderson presented the thought of forward security in the setting of mark to confine the harm of key introduction. The center thought is partitioning the entire lifetime of a private key into $\mathrm{T}$ discrete eras, with the end goal that the trade off of the private key for current era can't empower a foe to deliver substantial marks for past eras. Accordingly, Bellare and Miner gave formal meanings of forward-secure mark and exhibited functional arrangements. From that point forward, an extensive number of forward-secure signature schemeshas been proposed.

\subsection{Existing System}

Boneh and Franklin initially proposed a characteristic disavowal route for IBE. They affixed the present day and age to the ciphertext, and non-denied clients occasionally got private keys for each time period from the key authority.

Boldyreva, Goyal and Kumar acquainted a novel approach with accomplish effective denial. They utilized a parallel tree to oversee character with the end goal that their RIBE plot lessens the intricacy of key repudiation to logarithmic (rather than straight) in the greatest number of framework clients.

Subsequently, by utilizing the previously mentioned renouncement procedure, Libert and Vergnaud proposed an adaptively secure RIBE conspire in view of a variation ofWater's IBE plot.

Chen et al. built a RIBE scheme from lattices.

\subsubsection{Disadvantage of Existing System}


Unfortunately, existing arrangement is not versatile, since it requires the key specialist to perform direct work in the quantity of non-denied clients. Moreover, a safe channel is fundamental for the key expert and non-renounced clients to transmit new keys.

However, existing plan just accomplishes specific security.

This sort of revocation method can't avoid the plot of repudiated clients and malevolent non-renounced clients as pernicious non-denied clients can impart the overhaul key to those repudiated clients.

Furthermore, to overhaul the ciphertext, the key expert in their plan needs to keep up a table for every client to deliver the re-encryption key for every day and age, which fundamentally builds the key authority's workload.

\subsection{Proposed System}

It appears that the idea of revocable identity-based encryption (RIBE) may be a promising methodology that satisfies the previously mentioned security prerequisites for information sharing.

RIBE highlights an instrument that empowers a sender to affix the present day and age to the ciphertext with the end goal that the collector can decode the ciphertext just under the condition that he/she is not denied at that time period.

A RIBE-based information sharing system works as follows:

Step 1: The information supplier (e.g., David) first chooses the clients (e.g., Alice and Bob) who can share the information. At that point, David scrambles the information under the characters Alice and Bob, and transfers the ciphertext of the common information to the cloud server.

Step 2: When either Alice or Bob needs to get the common information, she or he can download and decrypt the comparing ciphertext. Be that as it may, for an unapproved client and the cloud server, the plaintext of the common information is not accessible.

Step 3: In some cases, e.g., Alice's approval gets lapsed, David can download the ciphertext of the mutual information, and afterward unscramble then-re-encode the common information to such an extent that Alice is kept from getting to the plaintext of the common information, and after that transfer the re-encrypted information to the cloud server once more.

\subsubsection{Advantages of Proposed System}


We give formal definitions to RS-IBE and its relating security model. The proposed plan can give classification and in reverse/forward2 mystery all the while. We demonstrate the security of the proposed scheme in the standard model, under the decisional $\ell$-Bilinear Diffie-Hellman Exponent $(\ell-\mathrm{BDHE})$ supposition. What's more, the proposed plan can withstand decryption key exposure.

The methodology of ciphertext upgrade just needs open data. Take note of that no past character based encryption plots in the writing can give this element. The extra calculation and capacity multifaceted nature, which are presented in by the mystery, is all upper limited by $\mathrm{O}(\log (\mathrm{T}) 2)$, where $\mathrm{T}$ is the aggregate number of eras.

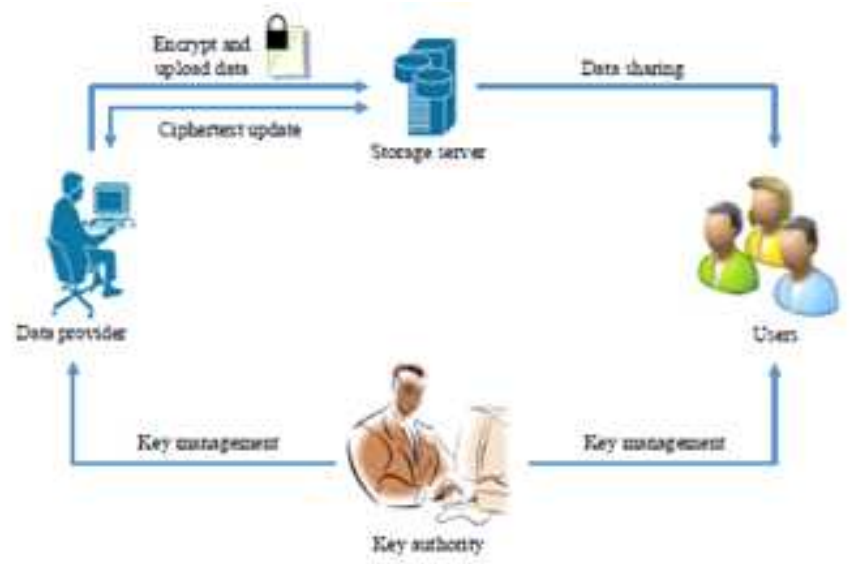

[Fig 1] System Architecture

\section{Conclusion}

Cloud computing brings incredible comfort for individuals. Especially, it superbly coordinates the expanded need of sharing information over the Internet. In this paper, to assemble a practical and secure information sharing framework in cloud computing, we proposed a thought called RS-IBE, which bolsters personality repudiation and ciphertext upgrade at the same time with the end goal that a denied client is kept from getting to beforehand shared information, and also along these lines shared information. Moreover, a solid development of RS-IBE is introduced. The proposed RS-IBE plan is demonstrated versatile secure in the standard model, under the decisional $\ell$-DBHE presumption. The examination comes about exhibit that our plan has favorable circumstances as far as productivity and usefulness, and in this manner is more attainable for functional applications. 


\section{References}

[1] L. M. Vaquero, L. Rodero-Merino, J. Caceres and M. Lindner, Abreak in the clouds: towards a cloud definition ACM SIGCOMMComputer Communication Review, (2008), Vol.39, No.1, pp.50-55,

[2] https://www.icloud.com/, March 23 (2014).

[3] http://www.windowsazure.com/, January 15 (2014).

[4] http://aws.amazon.com/s3/, June 7 (2014).

[5] K. Chard, K. Bubendorfer, S. Caton and O. F. Rana, Social cloudcomputing: A vision for socially motivated resource sharing Services Computing, IEEE Transactions on, (2012), Vol.5, No.4, pp.551-563.

[6] C. Wang, S. S. Chow, Q. Wang, K. Ren and W. Lou, Privacy preserving public auditing for secure cloud storage Computers, IEEE Transactions on, (2013), Vol.62, No.2, pp.362-375.

[7] G. Anthes, Security in the cloud, Communications of the ACM, (2010), Vol.53, No.11, pp.16-18.

[8] K. Yang and X. Jia, An efficient and secure dynamic auditing protocol for data storage in cloud computing Parallel and Distributed Systems, IEEE Transactions on, (2013), Vol.24, No.9, pp.1717-1726.

[9] B. Wang, B. Li and H. Li, Public auditing for shared data with efficient user revocation in the cloud in INFOCOM, 2013 Proceedings IEEE. IEEE, (2013), pp.2904-2912.

[10] S. Ruj, M. Stojmenovic and A. Nayak, Decentralized access control with anonymous authentication of data stored in clouds Parallel and Distributed Systems, IEEE Transactions on, (2014), Vol.25, No.2, pp.384-394.

[11] X. Huang, J. Liu, S. Tang, Y. Xiang, K. Liang, L. Xu and J. Zhou, Cost-effective authentic and anonymous data sharingwith forward security Computers, IEEE Transactions on, (2014), doi:10.1109/TC.2014.2315619.

[12] C. K. Chu, S. S. Chow, W. G.Tzeng, J. Zhou and R. H. Deng, Key-aggregate cryptosystem for scalable data sharing in cloudstorage Parallel and Distributed Systems, IEEE Transactions, (2014), Vol.25, No.2, pp.468-477.

[13] A. Shamir, Identity-based crypto systems and signature schemes in Advances in cryptology. Springer, (1985), pp.47-53.

[14] D. Boneh and M. Franklin, Identity-based encryption from theweil pairing, SIAM Journal on Computing, (2003), Vol.32, No.3, pp.586-615.

[15] S. Micali, Efficient certificate revocation Tech. Rep., (1996).

[16] W. Aiello, S. Lodha and R. Ostrovsky, Fast digital identity revocation in Advances in CryptologyCRYPTO 1998. Springer, (1998), pp.137-152.

[17] D. Naor, M. Naor and J. Lotspiech, Revocation and tracing schemes for stateless receivers in Advances in Cryptology - CRYPTO 2001. Springer, (2001), pp.41-62.

[18] C. Gentry, Certificate-based encryption and the certificate revocation problem in Advances in Cryptology- 
EUROCRYPT 2003.Springer, (2003), pp.272-293.

[19] V. Goyal, Certificate revocation using fine grained certificate space partitioning in Financial Cryptography and Data Security. Springer, (2007), pp.247-259.

[20] A. Boldyreva, V. Goyal and V. Kumar, Identity-based encryption with efficient revocation, Proceedings of the 15th ACM conference on Computer and communications security. ACM, (2008), pp.417-426.

[21] B. Libert and D. Vergnaud, Adaptive-id secure revocable identity based encryption in Topics in Cryptology - CT-RSA 2009. Springer, (2009), pp.1-15.

[22] B. Libert and D. Vergnaud, Towards Black-Box Accountable Authority IBE with Short Ciphertexts and Private Keys, Cryptography - PKC2009. Springer, (2009), pp.235-255.

[23] J. Chen, H. W. Lim, S. Ling, H. Wang and K. Nguyen, Revocable identity-based encryption from lattices in Information Security and Privacy, Springer, (2012), pp.390-403.

[24] J. H. Seo and K. Emura, Revocable identity-based encryption revisited: Security model and construction in Public-Key Cryptography - PKC 2013. Springer, (2013), pp.216-234.

[25] J. H. Seo and K. Emura, Efficient Delegation of Key Generation and Revocation Functionalities in Identity-Based Encryption, Topics in Cryptology - CT-RSA 2013. Springer, (2013), pp.343-358.

[26] K. Liang, J. K. Liu, D. S. Wong and W. Susilo, An efficient cloud based revocable identity-based proxy re-encryption scheme for public clouds data sharing in Computer Security-ESORICS 2014.Springer, (2014), pp.257-272. 
Email Based Verification of Data Storage in Cloud Computing

(This page is empty intentionally) 